Pacific Journal of Mathematic 


\title{
REARRANGEMENTS OF FUNCTIONS ON THE RING OF INTEGERS OF A $p$-SERIES FIELD
}

Benjamin B. Wells, JR.

\begin{abstract}
We show that every continuous function on the ring of integers of a $p$-series field has a rearrangement that has absolutely convergent Fourier series.
\end{abstract}

I. Introduction. Let $p$ be a rational prime fixed throughout. $K$ will denote the $p$-series field of formal Laurent series in one variable with finite principal part and with coefficients in $G F(p)$, Thus, an element $x \in K$ has representation as

$$
x=\sum a_{j} \mathfrak{p}^{j} \quad\left(a_{j}=0,1, \cdots, p-1\right)
$$

and $a_{j}=0$ for $j$ sufficiently small. Addition and multiplication are defined by the usual formal sums and products of Laurent series.

The field $K$ is topologized by taking as a basis the sets

$$
V_{x, k}=\left\{\sum b_{j} \mathfrak{p}^{j}: b_{j}=a_{j}, j<k\right\}
$$

where $x=\sum a_{j} \mathfrak{p}^{j}$. With this topology, $K$ is locally compact, totally disconnected and nondiscrete.

The ring of integers $\mathfrak{O}=\left\{x: x=\sum_{j=0}^{\infty} a_{j} \mathfrak{p}^{j}\right\}$ is the unique maximal compact subring of $K$. Let $d x$ denote Haar measure on $K$ derived from the additive structure and normalized so that $\mathfrak{D}$ has measure 1 .

As a locally compact abelian group, $\mathfrak{O}$ has a Pontryagin dual $\hat{\mathfrak{O}}$ that may be identified with $K / \mathfrak{O}$. We choose the representatives of the form

$$
\sum_{-1}^{-\nu} r_{j} \mathfrak{p}^{j} \quad\left(r_{j}=0,1, \cdots, p-1\right)
$$

and use the lexicographic ordering to match the characters $\chi_{t}$ to the nonnegative integers. Of course, if $\chi$ is a continuous unitary character of $K^{+}$, then $\chi(x)$ is a $p$ th root of unity for all $x \in K$.

If $f$ is an integrable function on $\mathfrak{D}$, its Fourier coefficients are given by

$$
\widehat{f}(t)=\int_{0} f(x) \bar{\chi}_{t}(x) d x \quad(t=0,1, \cdots) .
$$

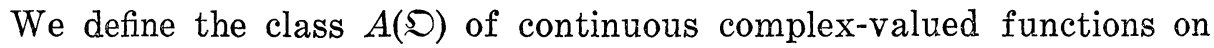
$\subseteq$ as those functions $f$ for which the quantity

$$
\sum_{t=0}^{\infty}|\hat{f}(t)|
$$


is finite. Under the pointwise operations $A(\mathfrak{D})$ is an algebra; it is, in fact, a Banach algebra with the above taken as the norm of $f$.

Suppose that $h$ is a homeomorphism of $\mathfrak{D}$, and that $f$ and $g$ are two functions on $\mathfrak{O}$ related by

$$
g=f \circ h .
$$

Then $g$ is said to be a rearrangement of $f$. N. Lusin asked whether every continuous function on the circle group has a rearrangement that has absolutely convergent Fourier series (see [4] p. 8). This question is still open; however, see [3] for the best known result. Here we prove the following.

THEOREM. Every continuous function $f$ on $\mathfrak{D}$ has a rearrangement $g$ that has absolutely convergent Fourier series.

It should be noted that the setting of the theorem contains as a special case $(p=2)$ the classical dyadic group $2^{\omega}$.

II. Preliminaries. The principal ideal in $\mathfrak{D}$ generated by $\mathfrak{p}, \mathfrak{P}$, is the unique maximal ideal in $\mathfrak{O}$. There is a non-archimedian valuation $|\cdot|$ on $K$ given by setting

$$
|\mathfrak{p}|=p^{-1} .
$$

$|\cdot|$ satisfies $|x+y| \leqq \operatorname{Max}\{|x|,|y|\}(x, y \in K)$, and therefore defines a metric on $K$. The topology induced by this metric coincides with that defined earlier.

The fractional ideals $\mathfrak{p}^{\nu}$ are given by

$$
\mathfrak{S}^{\nu}=\left\{x:|x| \leqq p^{-\nu}\right\} \text {. }
$$

Now for each $\nu, \mathfrak{O}$ decomposes into $p^{\nu}$ pairwise disjoint spheres $\omega(\nu, j)$, each of measure $p^{-\nu}$,

$$
\omega(\nu, j)=x_{j}+\mathfrak{P}^{\nu} \quad\left(j=1,2, \cdots, p^{\nu}\right) .
$$

We assume that the $x_{j}$ are ordered lexicographically. Thus, consecutive blocks of length $p^{\nu-1}$ have the same coefficient of the $\mathfrak{S}^{0}$ term, consecutive blocks of length $p^{\nu-2}$ have the same coefficient of the $\mathfrak{S}^{6}$ and $\mathfrak{P}^{1}$ terms, etc.

Consequently, we have the containments

$$
\omega(\nu+1, j) \subset \omega(\nu, k), \quad((k-1) p+1 \leqq j \leqq k p) .
$$

In our construction of a homeomorphism of $\subseteq$ it will be necessary to make repeated use of the fact that two compact, totally discon- 
nected, metrizable, and perfect spaces are homeomorphic (see [1] p. 97).

From now on, since the prime number $p$ will frequently occur exponentiated and subscripted, for typographical reasons we shall write $p(\nu)$ for $p^{\nu}$.

III. Lemma. Suppose that $g$ is a continuous complex-valued function defined on $\mathfrak{O}$. Then $g$ is an A-function if the series whose $n$th term $(n=0,1,2, \cdots)$ is given by

$$
p(n)(p-1) \sum_{i=1}^{p(n)} \min _{b_{i}} \int_{\omega(n, i)}\left|g(x)-b_{i}\right| d x
$$

is convergent. If $M$ denotes the sum of this series, then $\|g\|_{A} \leqq$ $M+\|g\|_{\infty}$.

Proof. Suppose that $g$ is locally constant on $\mathfrak{D}$ and takes the value $a_{j}$ on $\omega(\nu, j),(j=1, \cdots, p(\nu))$. Then

$$
\hat{g}(t)=\int_{0} g \bar{\chi}_{t} d x=\sum_{k=1}^{p(\nu)} a_{k} \int_{\omega(\nu, k)} \bar{\chi}_{t} d x .
$$

Now, if $t \geqq p(\nu)$, it follows from the orthogonality relatians (see [2] p. 613) that $\hat{g}(t)=0$. Suppose that $0 \leqq t \leqq p(\nu)$, and therefore that $\chi_{t}$ is a character identified with a representative of $K / \mathfrak{O}$ of the form

$$
\sum_{j=-1}^{-\nu} r_{j} \mathfrak{p}^{j} \quad\left(r_{j}=0,1, \cdots, p-1\right) .
$$

There are $p(n)(p-1)$ characters corresponding to the representatives (3) with $r_{j}=0, j<-n-2, r_{-n-1} \neq 0,-1<n<\nu$.

Consider the sum

$$
\sum_{t=0}^{p(\nu)-1}|\hat{g}(t)|
$$

In order to estimate (4), let $\chi_{t}$ be a character corresponding to (3) with $r_{-\nu}=r_{-\nu+1}=\cdots r_{-n-2}=0, r_{-n-1} \neq 0$, and $-1<n$. From (2) we see that

$$
\begin{aligned}
p(\nu) \hat{g}(t)= & \left\{A_{1}^{1} z^{1+q_{1}}+\cdots+A_{p}^{1} z^{p+q_{1}}\right\} \\
& +\cdots \\
& +\left\{A_{1}^{p(n)} z_{p(n)}^{1+q}+\cdots+A_{p}^{p(n)} z_{p(n)}^{p+q}\right\},
\end{aligned}
$$

where the $A$ 's are the sums of consecutive blocks of the $a$ 's of length $p(\nu-(n+1))$.

$$
\begin{aligned}
& A_{1}^{1}=a_{1}+\cdots+a_{p(\nu-(n+1))} \\
& \cdots \\
& A_{p}^{p(n)}=a_{p(\nu)-p(\nu-(n+1))+1}+\cdots+a_{p(\nu)} .
\end{aligned}
$$


Furthermore, $z \neq 1$ is a $p$ th root of unity, and $q_{1}, \cdots, q_{p(n)}$ are positive integers which depend on $\chi_{t}$.

Since the sum of $p$ successive powers of of a $p$ th root of unity $\neq 1$ is zero, we see that for arbitrary complex numbers $b_{1}, \cdots, b_{p(n)}$

$$
\begin{aligned}
\hat{g}(t)= & \hat{g}(t)-p(\nu-(n+1)) b_{1}\left(z+\cdots+z^{p}\right)- \\
& \cdots-p(\nu-(n+1)) b_{p(n)}\left(z+\cdots+z^{p}\right) .
\end{aligned}
$$

Combining (5) and (6) and applying the triangle inequality, we see that

$$
|\widehat{g}(t)| \leqq\left\{\sum_{k=1}^{p(\nu-n)}\left|a_{k}-b_{1}\right|+\cdots+{ }_{k=p(\nu)-p(\nu-n)+1} \sum_{k(\nu)}\left|a_{k}-b_{p(n)}\right|\right\} 1 / p(\nu) .
$$

However, the right hand side of (7) is just

$$
\sum_{i=1}^{p(n)} \int_{\omega(n, i)}\left|g(x)-b_{i}\right| d x
$$

Since there are $p(n)(p-1)$ characters $\chi_{t}$ of the type under consideration, the lemma is proved in the case that $g$ is locally constant.

Now assume that $g$ is an arbitrary continuous function on $\mathfrak{D}$ which satisfies the hypothesis of the lemma. Let $N$ be a fixed positive integer, and approximate $g$ uniformly on $\mathfrak{D}$ by a sequence $g_{m}$ of locally constant continuous functions. Now, for every choice of integer $n$ and complex numbers $b_{j}(1 \leqq j \leqq p(n))$ we have

$$
\sum_{j=1}^{p(n)} \int_{\omega(n, j)}\left|g_{m}(x)-b_{j}\right| d x \longrightarrow \sum_{j=1}^{p(n)} \int_{\omega(n, j)}\left|g(x)-b_{j}\right| d x
$$

as $m \rightarrow \infty$. Since the left hand side of (8) bounds $\left|\hat{g}_{m}(t)\right|$, where $\chi_{t}$ is a character corresponding to (3) with $r_{j}=0, j<-(n+1), r_{-n-1} \neq$ 0 , it follows that for arbitrary $\varepsilon>0$ that

$$
\sum_{t=0}^{N}\left|\widehat{g}_{m}(t)\right|<M+\|g\|_{\infty}+\varepsilon
$$

when $m$ is sufficiently large. Furthermore, since for each $t, \hat{g}_{m}(t) \rightarrow$ $\hat{g}(t)$ as $m \rightarrow \infty$, we conclude that

$$
\sum_{t=0}^{N}|\hat{g}(t)| \leqq M+\|g\|_{\infty}+\varepsilon .
$$

Since $N$ and $\varepsilon$ are arbitrary, the lemma is proved.

IV. Proof of the theorem. Suppose without loss of generality that $\|f\|_{\infty}=1$; we show how to construct a homeomorphism $h$ of $\mathfrak{D}$ such that $g=f \circ h$ satisfies the hypothesis of the lemma. Thus we will have rearrangement of $f$ whose Fourier series converges absolutely. 
We shall construct $h$ as a limit of homeomorphisms $H_{n}$

$$
h=\lim _{n} H_{n}
$$

where $H_{n}$ is a composition of $n$ homeomorphisms of $\mathfrak{O}, h_{1} \circ h_{2} \circ \cdots \circ h_{n}$. We begin by describing the construction of the $h$ 's.

For $U \subset \mathfrak{O}$, it will be convenient to use the following notation

$$
O_{f}(U)=\sup _{x, y \in U}|f(x)-f(y)| .
$$

The quantity $O_{f}(U)$ is referred to as the oscillation of $f$ on $U$.

Choose a partition of $\mathfrak{O}$ consisting of mutually disjoint, nonvoid, open and closed sets $U_{j}(1 \leqq j \leqq p+1)$ such that the oscillation of $f$ on the union of the $U_{j}(1 \leqq j \leqq p)$ is less than or equal $1 / p(3)$. Thus,

$$
O_{f}\left(\bigcup_{j=1}^{p} U_{j}\right) \leqq 1 / p(3) .
$$

Then take $h_{1}$ to be a homeomorphism of $\mathfrak{O}$ satisfying the following requirements

$$
\begin{aligned}
& h_{1}(\omega(1, j))=U_{j} \quad(1 \leqq j \leqq p-1) \\
& h_{1}\left(\omega(1, p) \backslash \omega(3, p(3))=U_{p}\right. \\
& h_{1}\left(\omega(3, p(3))=U_{p+1} .\right.
\end{aligned}
$$

Now suppose that $h_{1}, \cdots, h_{n-1}$ are homeomorphisms of $\mathfrak{D}$ that have been defined. Set $H_{n-1}=h_{1} \circ h_{2} \circ \cdots \circ h_{n-1}$.

We now turn to the definition of $h_{n}$. For $i=1, \cdots, p(n-1)$ let $U_{i, j}(1 \leqq j \leqq p+1)$ denote a partition of $\omega(n-1, i)$ into open and closed sets such that the following are satisfied.

$$
\begin{gathered}
O_{f^{\circ} H_{n-1}}\left(\bigcup_{j=1}^{p} U_{i, j}\right) \leqq 1 / p(2 n+1) \quad(i=1,2, \cdots, p(n-1)) \\
\omega\left(3(n-1), i p(2(n-1)+1) \subset U_{i p, p+1} \quad(i=1,2, \cdots, p(n-2)) .\right.
\end{gathered}
$$

Then take $h_{n}$ to be a homeomorphism of $\mathfrak{D}$ satisfying the following requirements $(i=1,2, \cdots, p(n-1))$

$$
\begin{gathered}
h_{n}(\omega(n, k))=U_{i, j} \quad(k=(i-1) p+j, 1 \leqq j \leqq p-1) \\
h_{n}(\omega(n, i p) \backslash \omega(3 n, i p(2 n+1)))=U_{i, p} \\
h_{n}\left(\omega(3 n, i p(2 n+1))=U_{i, p+1} .\right.
\end{gathered}
$$

Finally, we set $H_{n}=H_{n-1} \circ h_{n}$.

First, we observe that

$$
h_{n} \omega(n-1, i)=\omega(n-1, i) \quad(i=1,2, \cdots, p(n-1)) .
$$


From (14) we see that for every neighborhood $V$ of $0, h_{n}(x)$ and $h_{n}^{-1}(x)$ belong to $V+x$ for $n$ sufficiently large. From this follows the existence of the limits

$$
\lim _{n} H_{n}=h, \lim _{n} H_{n}^{-1}=h^{-1} .
$$

Again, from (14) the continuity of $h$ is clear. Therefore $h$ is a welldefined homeomorphism of $\mathfrak{O}$.

Set $g=f \circ h$. The function $g$ is then our rearrangement of $f$, and it remains to check that series described in the lemma is convergent.

Now, the inequalities

$$
\begin{aligned}
O_{f^{\circ} H_{n}}(\omega(n, j)) \leqq 1 / p(2 n+1) \quad(j & =(i-1) p+k, 1 \leqq k \leqq p-1, \\
i & =1, \cdots, p(n-1))
\end{aligned}
$$

follow immediately from (9) and (11). Sucessive application of (14) therefore yields

$$
\begin{aligned}
O_{g}(\omega(n, j)) \leqq 1 / p(2 n+1) \quad(j & =(i-1) p+k, 1 \leqq k \leqq p-1 \\
i & =1, \cdots, p(n-1))
\end{aligned}
$$

The inequalities

$$
\begin{aligned}
O_{f \circ H n}(\omega(n, i p) \backslash \omega(3 n, i p(2 n+1)) \leqq & 1 / p(2 n+1) \\
& (i=1,2, \cdots, p(n-1))
\end{aligned}
$$

follow from (9) and (12). Relation (10) (with $n-1$ replaced by $n$ ) and the fact that $\omega(3 n, i p(2 n+1)) \supset \omega\left(3(n+1), i^{\prime} p(2(n+1)+1)\right)$, where $i^{\prime}=i p$, imply that (16) holds with $H_{n}$ replaced by $H_{n+1}$. This last step may be successively repeated to obtain

$$
O_{f^{\circ} H_{m}}(\omega(n, i p) \backslash \omega(3 n, i p(2 n+1)) \leqq 1 / p(2 n+1) \quad(n \leqq m) .
$$

However, since $f \circ H_{m}$ tends uniformly to $g$ we see that

$$
O_{g}(\omega(n, i p) \backslash \omega(3 n, i p(2 n+1)) \leqq 1 / p(2 n+1) .
$$

From (15) and the fact that the measure of $\omega(n, j)$ is $p(-n)$ we obtain the inequalities

$$
\begin{aligned}
\min _{b_{j}} \int_{\omega(n, j)}\left|g(x)-b_{j}\right| d x \leqq 1 /\{p(2 n+1) p(n)\} \\
\quad(j=(i-1) p+k, 1 \leqq k \leqq p-1, i=1, \cdots, p(n-1)) .
\end{aligned}
$$

From (18) we deduce that

$$
\begin{array}{r}
\min _{b_{j}} \int_{\omega(n, j)}\left|g(x)-b_{j}\right| d x \leqq 1 /\{p(2 n+1) p(n)\}+2 / p(3 n) \\
(j=i p, i=1, \cdots, p(n-1)) .
\end{array}
$$


We consider now the $n$th term of the series described in the lemma. Combining (19) and (20) we obtain the inequality

$$
\begin{aligned}
p(n)(p-1) \sum_{j=1}^{p(n)} \min _{b_{j}} \int_{\omega(n, j)}\left|g(x)-b_{j}\right| d x \leqq & p(n)(p-1)\{1 / p(2 n+1) \\
& +2 p(n-1) / p(3 n)\} \\
& \leqq \\
& 3 / p(n) .
\end{aligned}
$$

Therefore, the series of the lemma is convergent. The proof of the theorem is now complete.

\section{REFERENCES}

1. J. C. Hocking and G. S. Young, Topology, Addison-Wesley (1961) Reading, Mass.

2. R. A. Hunt and M. H. Taibleson, Almost everywhere convergence of Fourier series on the ring of integers of a local field, SIAM J. Math. Anal., 2 (1971), 607-625.

3. J.-P. Kahane, Sur les réarrangements de fonctions de la classe A, Studia Math., 31 (1968), 287-293.

4. - Séries, de Fouier Absolument Convergentes, Ergebnisse, Band 50, SpringerVerlag, 1970.

5. M. H. Taibleson, Fourier series on the ring of integers in a p-series field, Bull. Amer. Math. Soc., 73 (1967), 623-629.

Received October 10, 1974.

University of Hawail, Honolulu 



\section{PACIFIC JOURNAL OF MATHEMATICS}

\section{EDITORS}

RICHARD ARENS (Managing Editor) University of California

Los Angeles, California 90024

R. A. Beaumont

University of Washington

Seattle, Washington 98105
J. DUGUNDJI

Department of Mathematics University of Southern Californı

Los Angeles, California 90007

D. Gilbarg and J. Milgram

Stanford University

Stanford, California 94305

\section{ASSOCIATE EDITORS}
E. F. BECKENBACH
B. H. NeumanN
F. WOLF
K. YosHIDA

\section{SUPPORTING INSTITUTIONS}

UNIVERSITY OF BRITISH COLUMBIA

CALIFORNIA INSTITUTE OF TECHNOLOGY

UNIVERSITY OF CALIFORNIA

MONTANA STATE UNIVERSITY

UNIVERSITY OF NEVADA

NEW MEXICO STATE UNIVERSITY

OREGON STATE UNIVERSITY

UNIVERSITY OF OREGON

OSAKA UNIVERSITY
UNIVERSITY OF SOUTHERN CALIFORNIA

STANFORD UNIVERSITY

UNIVERSITY OF TOKYO

UNIVERSITY OF UTAH

WASHINGTON STATE UNIVERSITY

UNIVERSITY OF WASHINGTON

* * * *

AMERICAN MATHEMATICAL SOCIETY

NAVAL WEAPONS CENTER 


\section{Pacific Journal of Mathematics \\ Vol. 65, No. $1 \quad$ September, 1976}

David Lee Armacost, Compactly cogenerated LCA groups ............. 1

Sun Man Chang, On continuous image averaging of probability measures ...... 13

J. Chidambaraswamy, Generalized Dedekind $\psi$-functions with respect to a

polynomial. II................................... 19

Freddy Delbaen, The Dunford-Pettis property for certain uniform algebras ..... 29

Robert Benjamin Feinberg, Faithful distributive modules over incidence

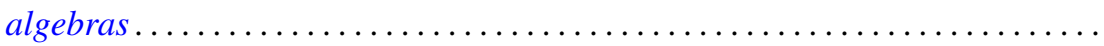

Paul Froeschl, Chained rings . . . . . . . . . . . . . . . . . . . .

John Brady Garnett and Anthony G. O'Farrell, Sobolev approximation by a sum

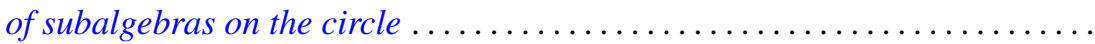

Hugh M. Hilden, José M. Montesinos and Thomas Lusk Thickstun, Closed

oriented 3-manifolds as 3-fold branched coverings of $S^{3}$ of special type.....

Atsushi Inoue, On a class of unbounded operator algebras ................

Peter Kleinschmidt, On facets with non-arbitrary shapes.

Narendrakumar Ramanlal Ladhawala, Absolute summability of Walsh-Fourier

series

Howard Wilson Lambert, Links which are unknottable by maps . . . . . . . . . . .

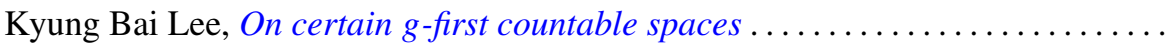

Richard Ira Loebl, A Hahn decomposition for linear maps .................

Moshe Marcus and Victor Julius Mizel, A characterization of functionals on $W_{1}^{p}$ possessing autonomous kernels. I . .

James Miller, Subordinating factor sequences and convex functions of several

variables.

Keith Pierce, Amalgamated sums of abelian l-groups ...

Jonathan Rosenberg, The $C^{*}$-algebras of some real and $p$-adic solvable

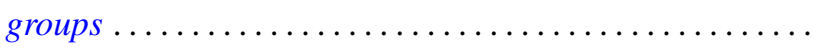

Hugo Rossi and Michele Vergne, Group representations on Hilbert spaces defined

in terms of $\partial_{b}$-cohomology on the Silov boundary of a Siegel domain . .

Mary Elizabeth Schaps, Nonsingular deformations of a determinantal

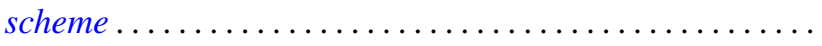

S. R. Singh, Some convergence properties of the Bubnov-Galerkin method...

Peggy Strait, Level crossing probabilities for a multi-parameter Brownian

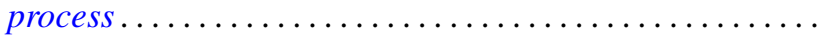

Robert M. Tardiff, Topologies for probabilistic metric spaces .

Benjamin Baxter Wells, Jr., Rearrangements of functions on the ring of integers of

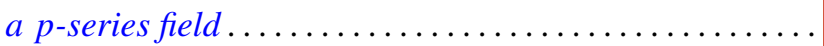

Robert Francis Wheeler, Well-behaved and totally bounded approximate identities for $C_{0}(X)$.

Delores Arletta Williams, Gauss sums and integral quadratic forms over local

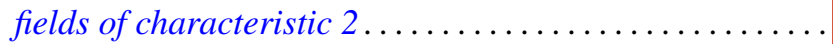

John Yuan, On the construction of one-parameter semigroups in topological 